\title{
Hardware Acceleration of Modern Data Management
}

\author{
Kai-Uwe Sattler ${ }^{(凶)}$ \\ TU Ilmenau, Ilmenau, Germany \\ kus@tu-ilmenau.de
}

\begin{abstract}
Over the past thirty years, database management systems have been established as one of the most successful software concepts. In todays business environment they constitute the centerpiece of almost all critical IT systems. The reasons for this success are manyfold. On the one hand, such systems provide abstractions hiding the details of underlying hardware or operating systems layers. On the other hand, database management systems are ACID compliant, which enables them to represent an accurate picture of a real world scenario, and ensures correctness of the managed data.

However, the currently used database concepts and systems are not well prepared to support emerging application domains such as eSciences, Industry 4.0, Internet of Things or Digital Humanities. Furthermore, volume, variety, veracity as well as velocity of data caused by ubiquitous sensors have to be mastered by massive scalability and online processing by providing traditional qualities of database systems like consistency, isolation and descriptive query languages. At the same time, current and future hardware trends provide new opportunities such as many-core CPUs, co-processors like GPU and FPGA, novel storage technologies like NVRAM and SSD as well as high-speed networks provide new opportunities.

In this talk we present our research results for the use of modern hardware architectures for data management. We discuss the design of data structures for persistent memory and the use of accelerators like GPU and FPGA for database operations.
\end{abstract}

